\section{Cahiers de Narratologie}

Analyse et théorie narratives

$15 \mid 2008$

Récits et genres historiques

\title{
Les récits historiques du Futurisme, ou l'histoire sans passé de l'avant-garde
}

Serge Milan

\section{(2) OpenEdition}

Journals

Édition électronique

URL : http://journals.openedition.org/narratologie/849

DOI : $10.4000 /$ narratologie.849

ISSN : 1765-307X

Éditeur

LIRCES

Référence électronique

Serge Milan, «Les récits historiques du Futurisme, ou l'histoire sans passé de l'avant-garde », Cahiers de Narratologie [En ligne], 15 | 2008, mis en ligne le 20 décembre 2008, consulté le 03 mai 2019. URL http://journals.openedition.org/narratologie/849; DOI : 10.4000/narratologie.849

Ce document a été généré automatiquement le 3 mai 2019.

Article L.111-1 du Code de la propriété intellectuelle. 


\title{
Les récits historiques du Futurisme, ou l'histoire sans passé de l'avant- garde
}

\author{
Serge Milan
}

1 Du point de vue des jeunes artistes qui constituent à partir de 1909 le mouvement d'avant-garde futuriste italien, les problèmes posés par l'Histoire dans les récits de fiction semblent être sans objet: les déclarations programmatiques contenues dans les premiers articles et manifestes du poète Filippo Tommaso Marinetti, le fondateur du Futurisme et son principal idéologue, imposent une poétique a priori étrangère au genre historique.

2 C'est qu'il s'agit avant tout précisément de libérer l'Italie, la "terre des morts" des Romantiques, de la tyrannie "du culte d'un passé trop imité et trop vénéré". Ainsi, Marinetti préconise une poésie "libre, émancipée de tous ses jougs traditionnels, et rythmée par la symphonie des assemblées, des usines, des automobiles et aéroplanes". Le Futurisme exaltera donc la modernité sous toutes ses formes, notamment selon le dernier point normatif du manifeste de fondation paru le 11 janvier 1909 dans Le Figaro:

Nous chanterons les grandes foules agitées par le travail, le plaisir ou la révolte: nous chanterons les ressacs multicolores et polyphoniques des révolutions dans les capitales modernes; la vibration nocturne des arsenaux et des chantiers sous leurs violentes lunes électriques; les gares gloutonnes avaleuses de serpents qui fument; les usines suspendues aux nuages par les ficelles de leurs fumées; les ponts aux bonds de gymnastes lancés sur la coutellerie diabolique des fleuves ensoleillés; les paquebots aventureux flairant l'horizon; les locomotives au grand poitrail qui piaffent sur les rails, tels d'énormes chevaux d'acier bridés de longs tuyaux, et le vol glissant des aéroplanes, dont l'hélice a des claquements de drapeau et des applaudissements de foule enthousiaste.

La "grande esthétique futuriste" vouée à "libérer l'Italie de sa fétide gangrène de professeurs, d'archéologues, de cicérones et d'antiquaires", qui veut raser et reconstruire une Venise et une Florence industrielles, qui "crache sur l'autel de l'Art avec un A majuscule" et veut substituer aux "sanglots nostalgiques et mélancoliques" du romantisme et à l'exotisme symboliste la "physico-folie" du café-théâtre et la Religion- 
morale de la vitesse ne semble alors guère laisser de place au genre historique, quelle que puisse être la discipline artistique envisagée. Et en effet, alors que le mouvement futuriste se structure par secteurs disciplinaires de plus en plus nombreux à partir du ralliement de peintres, de musiciens, d'architectes et d'autres artistes, les manifestes et les programmes confirment des intentions esthétiques et poétiques peu affines avec un genre dont l'objet principal est précisément la re-présentation des temps anciens.

Les nouvelles conditions de vie dans lesquelles nous vivons ont créé une infinité d'éléments naturels entièrement nouveaux, qui n'ont jamais fait leur entrée dans le domaine de l'art, et pour lesquels les futuristes se proposent de découvrir - à tout prix ! - de nouveaux moyens d'expression. ${ }^{1}$

Umberto Boccioni, le grand peintre qui fut avec Marinetti le promoteur et le théoricien du premier Futurisme, définit lui aussi un programme qui semble exclure pour les arts plastiques toute possibilité de représentation historique. Ces "primitifs d'une nouvelle sensibilité", comme se définissent les peintres futuristes, excluront de fait le genre historique de leur production, et ce pour la durée de vie entière du mouvement, une trentaine d'années environ; l'on peut par ailleurs remarquer que ce choix sera également celui de toutes les avant-gardes du $\mathrm{XX}^{\text {ème }}$ siècle, à quelques rares et iconoclastes exceptions près.

D'un point de vue littéraire, le constat est également fort rapidement fait: aucune œuvre poétique, aucun roman, aucune œuvre théâtrale futuriste parmi les dizaines publiées chez les différents éditeurs futuristes milanais, florentins ou romains, n'est une fiction historique; inutile de préciser que les œuvres graphiques, musicales, cinématographiques, les créations décoratives, vestimentaires, scénographiques, culinaires, pyrotechniques, tactiles, aérosculpturales, polymatérielles ou autres sont toutes immunes de quelque référence explicite que ce soit à l'histoire et au passé, et ces mots de Giovanni Papini, futuriste florentin et directeur de la grande revue d'avant-garde Lacerba, semblent résumer jusque dans l'injure (et dans la lignée du manifeste L'antitradition futuriste de Guillaume Apollinaire), le rejet en bloc du passé pour ces jeunes artistes:

Les futuristes ne peuvent nier le passé pour la simple raison que LE PASSÉ N'EXISTE PAS, et qu'on ne peut nier l'inexistant. Ils nient, répudient, refusent les idées stupides, les peurs idiotes, les idéaux débiles qui existent aujourd'hui dans les têtes des hommes vivants et présents. Ils comparent le passé à la lumière lointaines de ces étoiles qui disparurent des cieux il y a des siècles, et dont les rayons tardifs illuminent encore nos nuits. [...] les hommes ont une tendance ignoble à reconnaître la grandeur uniquement dans les œuvres ou les âmes lointaines. Ils n'attribuent de la valeur qu'à ce qui n'existe plus [...] Nous pensons que cette façon de faire est une ignoble infamie. Et nous protestons jusqu'à notre dernier souffle. Et puisque ces gens - critiques usuriers et artistes perroquets traditionalistes appellent cette injustice imbécile culte du passé, nous autres, à ce faux passé, et à ce présent mortifiant et geôlier, dirons éternellement MERDE. ${ }^{2}$

Ces différents constats, et ces quelques extraits de manifestes et articles des années Dix qui semblent réduire à néant toute recherche dans le domaine des avant-garde pour ce qui est du récit historique, appellent néanmoins, semble-t-il, un approfondissement et une réserve.

7 Pour ce qui est de l'approfondissement, il nous a semblé digne d'intérêt de revenir malgré tout sur le refus du genre historique par le Futurisme en examinant plus en détail les manifestes qui concernent en particulier le théâtre, forme exemplaire en ce qu'elle a donné lieu à des indication normatives très précises permettant de cerner au plus près le 
rapport entre le projet avant-gardiste et le genre historique, que l'on se place du point de vue de la fiction ou des études historiques.

Quant à la réserve, elle est de taille et concerne quatre ouvrages majeurs de Marinetti luimême, écrits entre 1915 et 1929, dans lesquels le genre narratif où domine la référence historique est prééminent, sans que cela ne surprenne un lecteur pourtant au fait de la révolte futuriste contre le passé sous toutes ses formes.

9 Mais avant de nous engager plus avant sur ces chemins, nous souhaitions rappeler comment et en quoi le rapport à l'histoire et au passé constituent précisément le problème constitutif de l'avant-garde futuriste, et plus généralement des avant-gardes qui marquèrent de façon si caractéristique les arts du siècle passé: nous le ferons en examinant une notion et une métaphore stratégiques dans la constitution idéologique du mouvement.

I.La guerre contre les morts : Futurismo contra passatismo

10 La métaphore de la guerre aux morts et aux moribonds utilisée dans les manifestes marinettiens du début des années Dix constitue sans aucun doute une marque stylistique inimitable du mouvement futuriste naissant: davantage même que les déclarations antiautrichiennes de Marinetti, elle canalise l'énergie polémique de ces premiers textes et leur violence déjà inouïe.

Que peut-on bien trouver dans un vieux tableau si ce n'est la contorsion pénible de l'artiste s'efforçant de briser les barrières infranchissables à son désir d'exprimer entièrement son rêve?

Admirer un vieux tableau, c'est verser notre sensibilité dans une urne funéraire, au lieu de la lancer en avant par jets violents de création et d'action. Voulez-vous donc gâcher ainsi vos meilleures forces dans une admiration inutile du passé, dont vous sortez forcément épuisés, amoindris, piétinés?

En vérité, la fréquentation quotidienne des musées, des bibliothèques et des académies (ces cimetières d'efforts perdus, ces calvaires de rêves crucifiés, ces registres d'élans brisés!...) est pour les artistes ce qu'est la tutelle prolongée des parents pour des jeunes gens intelligents, ivres de leur talent et de leur volonté ambitieuse.

Pour des moribonds, des invalides et des prisonniers, passe encore. C'est peut-être un baume à leurs blessures que l'admirable passé, du moment que l'avenir leur est interdit... Mais nous n'en voulons pas, nous les jeunes, les forts et les vivants futuristes! ${ }^{3}$

11 L'un des passages les plus dignes d'intérêt de Fondation et Manifeste du Futurisme, précisément celui où Marinetti définit pour la première fois les futuristes, confirme que la constitution du mouvement est principalement liée à une problématique d'ordre éthique, et que l'esthétique et la connaissance du passé envisagées en tant que milieu (ambiente, dans les versions italiennes des manifestes) sont intimement liées à cette problématique.

12 À la galerie d'œuvres exposées comme autant de loculi où se débattent des morts-vivants encore prêts à s'entredéchirer, répondent ici les "moribonds, invalides [et] prisonniers" du paragraphe suivant, auxquels "l'avenir est interdit". C'est entre ce passé qui n'en finit pas et ce futur impossible que se constitue la première personne futuriste, celle par laquelle "notre tristesse, notre courage fragile et notre inquiétude" se convertit un nouveau sujet: "nous, les jeunes, les forts et les vivants futuristes!".

13 Voilà la renaissance que Marinetti propose à son lecteur: il s'agit de prendre acte du fait que l'identification à l'artiste génial du passé se joue non pas au travers d'une forme - le vieux tableau comme urne funéraire - mais dans l'imitation d'un effort de destruction et 
d'expression. À défaut d'adhérer à cette identification dans l'acte, l'on risque une déperdition stérile de l'action créatrice, c'est-à-dire la captation de la sensibilité du vivant par le mort.

L'année 1910 verra la reprise de cette métaphore dans la plupart des textes et des discours marinettiens: le second manifeste Tuons le clair de lune!, la Lettre futuriste aux citoyens de Parme, le Discours aux habitants de Trieste, ou Contre l'Espagne passéiste sont structurés autour de cette unique métaphore ou y font allusion à plusieurs reprises. La rébellion au passé artistique est plus ciblée dans le manifeste des peintres de la même année, mais la continuité avec les poètes revendiquée au début du texte est assurée encore une fois par la même métaphore centrale, celle de la nation-cimetière ("pour les autres peuples, l'Italie est encore une terre des morts") utilisée également dans une Lettre circulaire aux journaux de 1910: "Grâce à ces jeunes artistes turbulents et audacieux, la Terre des morts sera bientôt le plus vivant des pays de la Terre"

Par ailleurs, les peintres reprennent les cibles marinettiennes presque telles quelles:

Hors d'ici, restaurateurs soudoyés de vieilles croûtes! Hors d'ici, archéologues malades de nécrophilie chronique! Hors d'ici, critiques, maquereaux complaisants!

Hors d'ici, académies goutteuses, professeurs alcooliques et ignorants! ${ }^{5}$

Ignominie, prostitution, canaille, paresse répugnante: les institutions qui opposent leur inertie au renouvellement de l'art sont injuriées selon une polémologie qui deviendra l'un des aspects essentiels des avant-gardes, tandis que le manifeste se clôt sur la nécessité encore revendiquée de se débarrasser, avant tout, des morts:

Que les morts soient enterrés dans les viscères les plus profondes de la terre! Que

l'on dégage le seuil du futur des momies! Place aux jeunes, aux violents, aux téméraires! ${ }^{6}$

17 C'est à partir de cette métaphore que la ville de Venise, "bordel" et "hôpital le plus triste du monde", sera qualifiée de "passéiste" dans le titre qui allait également devenir un point de repère dans "l'art de faire des manifestes", Contre Venise passéiste ${ }^{7}$. Il est vrai que par la suite, les jeunes artistes futuristes n'auront de cesse de redéfinir ce terme pour l'amener à contenir tout ce qui relève d'un rapport approfondi au passé, jusqu'à en faire une catégorie fondamentale de leur idéologie: la lutte qui verra s'affronter Futurisme et passéisme finira même par circonscrire le réel dans sa totalité (de la géopolitique à la psychologie, en passant par la morale et bien sûr, l'esthétique) submergeant toutes les autres catégories interprétatives dans un manichéisme volontairement radical et violent.

Cela étant, que ce passatismo naisse comme image de mort et comme catégorie culturelle regroupant les professions et les institutions de la culture et de l'éducation, mais aussi comme qualificatif de la ville qui représente presque par excellence le raffinement du goût et l'aventure sentimentale, n'est pas sans intérêt. L'adversaire contre lequel se structure l'idéologie du mouvement - car c'est bien dans cet affrontement que naît conceptuellement le Futurisme comme "autre chose" qu'une école littéraire, "autre chose" même que de l'art, pour reprendre un mot célèbre de Benedetto Croce - est avant tout ici un certain type de connaissance, à la fois modale et sectorielle: le passé historique et artistique vécu et assimilé au travers de l'éducation, des livres ou du «voyage en Italie» comme quête d'émotions culturelles et de mémoires pittoresques8.

19 C'est donc un certain type de savoir, qui relève de l'appropriation personnelle d'une mémoire collective, qui va véritablement armer le Futurisme et l'amener à la guerre: notre connaissance collective du passé, en ce qu'elle s'approfondit et se personnalise par 
la fréquentation des bibliothèques, des musées, ou des "villes vénérables". Que les futuristes fassent de tout cela une fréquentation de cimetières et de morts, et de ceux qui s'y livrent - artistes, lettrés et bourgeois - des "nécrophiles [...] fascinés par des momies", indique à quel point ils envisagent avec le plus grand intérêt le rapport de chacun à cette mémoire collective. Cette connaissance qu'ils veulent éradiquer, soulignons-le, n'est jamais de l'ordre de l'impersonnel, ou a contrario réservée au jeune artiste cherchant son art, mais elle relève au contraire de l'intime et du silencieux rapport de soi à soi qui se constitue en identité personnelle au moyen des différents miroirs dans lesquels l'on se contemple. Le combat contre le passéisme, c'est donc avant tout la redéfinition radicale de toutes les modalités du processus par lequel, pour le dire avec Nietzsche, l'on devient ce que l'on est ${ }^{9}$.

II. Théâtre et anthropogenèse

Une fois précisé le rapport constitutif de l'avant-garde au passé dans un cadre général, il est plus aisé de revenir aux réserves - au franc rejet, méprisant et indigné, en vérité - du Futurisme par rapport au genre historique en tant que tel:

Nous méprisons en art, et plus particulièrement au théâtre, tous types de reconstructions historiques, qu'elles tirent intérêt de la figure d'un héro ou d'une héroïne illustre (Néron, Jules César, Napoléon ou Francesca de Rimini), ou qu'elles reposent sur la suggestion exercée par l'inutile somptuosité des costumes et des décors du passé. Le drame moderne doit refléter en partie au moins le grand rêve futuriste qui émerge de notre vie contemporaine, exaspérée par les vitesses terrestres, marines et aériennes, et dominée par la vapeur et l'électricité. Il faut introduire au théâtre la sensation du règne de la Machine, les grands frissons qui agitent les foules, les nouveaux courants d'idées et les grandes découvertes de la science qui ont complètement transformé notre sensibilité et notre mentalité d'homme du vingtième siècle. ${ }^{10}$

Cet extrait du Manifeste des dramaturges (1911), renommé par Marinetti La volupté d'être sifflés, nous permet de resserrer notre propos.

Il explicite tout d'abord le rejet non plus seulement de la connaissance historique, mais bien celui de la fiction historique; et il en suggère des raisons qui nous permettent de conclure à la proximité de ces deux catégories pour l'avant-garde. On soulignera en effet l'importance, déjà remarquée plus haut, du rejet du décor et de tout ce qui en fait partie (les personnages historiques eux-mêmes sont d'ailleurs envisagés uniquement comme figure, figures imposantes et statuaires privées de vie). Or le décor, c'est précisément la négation d'une autre notion fondamentale, promue et chargée de sens par les textes futuristes déjà commentée ici: l'ambiente (parfois atmosfera), c'est-à-dire le milieu en tant qu'il participe de la vie et de la vibration universelle qui modèle, et qui est à son tour modelé, par le génie de l'artiste.

Par ailleurs, si l'on accepte provisoirement les conclusions de recherches personnelles et collectives concernant la nature profonde de l'avant-garde futuriste comme projet cosmogonique intégral (une Ricostruzione futurista dell'universo, pour reprendre le titre d'un manifeste crucial de 1915) et comme anthropogenèse eschatologique dans laquelle l'homme nouveau, que l'art total contribue à créer, substitue à la mémoire et à la raison une sensibilité des perceptions et des instincts qui lui autorise un accès direct à la nature ${ }^{11}$ qui l'entoure - si l'on accepte donc de considérer le Futurisme comme ce projet totalisant qu'il voulut être, les aspects gnoséologiques et anthropologiques que nous venons de souligner confèrent au théâtre, du point de vue des futuristes, une fonction exceptionnelle, explicitée par un autre manifeste de 1915, Il teatro futurista sintetico: 
En attendant notre grande guerre si souvent invoquée, nous autres futuristes alternons notre violente action antineutraliste dans les places et les Universités avec notre action artistique sur la sensibilité italienne, que nous voulons préparer à l'heure fatale du plus grand Danger. L'Italie se devra d'être intrépide, aussi acharnée que possible, élastique et rapide comme un escrimeur, indifférente aux coups comme un boxeur, impassible à l'annonce d'une victoire qui aurait coûté cinquante mille morts comme à l'annonce d'une défaite.

Pour que l'Italie apprenne à se décider avec la rapidité de l'éclair, à s'élancer, à soutenir tous les efforts et toute éventuelle déconvenue, les livres et les revues ne sont pas utiles. Ceux-ci n'intéressent que la minorité de personnes qui s'en occupent; ils sont plus ou moins ennuyeux, encombrants et lents, et ils ne peuvent que refroidir l'enthousiasme, briser l'élan et empoisonner de doutes un peuple qui combat. La Guerre, futurisme intensifié, nous impose de marcher et ne pas pourrir dans les bibliothèques et les salles de lecture. Nous croyons donc que l'on ne peut exercer une influence guerrière sur l'âme italienne, sinon par le théâtre. En effet $90 \%$ des Italiens vont au théatre, tandis que 10\% seulement lisent des livres et des revues. Mais il faut pour cela un théâtre futuriste, c'est-à-dire absolument opposé au théâtre passéiste, qui prolonge ses cortèges monotones et déprimant sur les scènes somnolentes italiennes. [...]

CONCLUSIONS :

1. Il faut détruire totalement la technique dont meurt le théâtre passéiste.

2. Il faut porter sur la scène toutes les découvertes et toutes les recherches, même les plus invraisemblables, les plus bizarres et les plus antithéâtrales qu'elles soient, que le génie artistique et la science font chaque jour à propos du subconscient, parmi les forces encore mal définies, dans l'abstraction pure, dans le cérébralisme pur, dans la fantaisie pure, le record et la folie physique du Music-hall. Ex. Ils viennent, le premier drame synthétique d'objets de Marinetti est de ce point de vue un nouveau filon de sensibilité théâtrale découvert par le futurisme.

3. Symphoniser la sensibilité du public, explorant et réveillant par tous les moyens possibles ses nerfs les plus assoupis ; détruire le préjugé de la rampe, en lançant des filets de sensations qui enveloppent la scène et le public ; l'action théâtrale doit envahir le parterre.

4. Fraterniser chaleureusement avec les acteurs comiques, qui sont peut-être les seuls penseurs qui sachent éviter tout effort culturel déformant.

5. Abolir la farce, le vaudeville, la comédie, le drame et la tragédie, pour créer à leur place les formes nombreuses du théâtre futuriste, telles que : les répliques en liberté, la simultanéité, la compénétration, le poème animé, l'hilarité dialoguée, l'acte négatif, la répartie répercutée, la discussion extralogique, la déformation synthétique, le soupirail scientifique.

6. Créer entre la foule et nous-mêmes, au moyen d'un contact continu, un courant de confiance sans respect, de façon à infuser dans les publics la vivacité dynamique d'une nouvelle théâtralité futuriste.

Voici les premières paroles sur le théâtre. [... ${ }^{12}$

Il est remarquable de constater à quel point le lien entre l'art et l'idéologie est exprimé dans ce manifeste théâtral de façon linéaire et concise: un art est ici examiné et jugé comparativement pour ses qualités propagandistes à l'exclusion de tout autre critère. La nécessité d'un théâtre futuriste apparaît dans le texte après une (approximative) démonstration statistique sur la portée médiatique respective du livre et de la scène théâtrale, alors même que la concurrence du cinématographe est évoquée. Il s'agit d' influenzare guerrescamente l'anima italiana mediante il teatro, un théâtre qui se devra pour cela d'être "synthétique, atechnique, dynamique, simultané, autonome, alogique, irréel". Cette option futuriste fondamentale pour un art "engagé" est intrinsèquement idéologique, en ce qu'elle suppose ce que Marinetti appelle ici et dans bien d'autres textes 
"les grandes foules", "les marées humaines", ou "le public" envisagé tour à tour comme multitude fascinante et canaille grégaire.

C'est que le nouveau théâtre futuriste exerce son pouvoir d'influence, dans la conception que s'en font ses créateurs, non pas au travers d'un discours idéologique diversement mis en scène, mais par le moyen même de ces nouvelles qualités formelles. Alors que toutes les caractéristiques du nouveau théâtre énumérées par le manifeste prennent le contrepied de la progression explicative et dramatique, du goût du public, de la logique et de la vraisemblance, ce sont le subcosciente et la fisicofollia (la "folie physique" des acrobates et des cabarétistes) qui sont présentés comme les colonnes portantes de l'art dramatique futuriste. Ceux-ci ne relèvent ni de l'émotivité, ni a fortiori du discursif, mais de la transmission, au sein d'un milieu dont la continuité énergétique semble être encore postulée a priori, d'un puissant mouvement chaotique ou vibratoire: l'azione scenica invaderà platea e spettatori, énonce radicalement la version italienne au point $n^{\circ} 3$.

La participation du public, la fin de la scène séparée et du spectacle encadré dans ses formes classiques au profit du contatto continuato et de la trasfusione seront approfondies non seulement dans les autres textes marinettiens sur le théâtre ${ }^{13}$, mais également dans la série des manifestes de Scenosintesi d'Enrico Prampolini. À la fin des années Vingt et au début des années Trente, celui-ci écrira des textes d'une grande créativité concernant l'organisation scénique de spectacles dramatiques dans la lignée de la collaboration de Giacomo Balla avec les Ballets Russes, centrés sur "l'acteur-espace", totalement abstraits et synesthétiques, et avec cette insistance toute particulière sur la nécessité de créer une "atmosphère scénique", c'est-à-dire une continuité organique de milieu capable de véhiculer un dynamisme "mécanique et spirituel" entre l'action scénographique et le public:

... la technique théâtrale s'oriente vers le dynamisme plastique de la vie contemporaine, l'action. Les principes fondamentaux qui animent l'atmosphère scénique sont l'essence même du spiritualisme, de l'esthétique et de l'art futuriste, soit: dynamisme, simultanéité et unité d'action entre homme et milieu. [...]

Nous, les futuristes, nous avons atteint et proclamé cette unité scénique en fusionnant l'élément homme et l'élément milieu dans une synthèse scénique vivante de l'action théâtrale. ${ }^{14}$

Plus généralement, en relisant les indications techniques de Prampolini et celles des "premières paroles sur le théâtre" de Marinetti (qui font écho aux "premières volontés des vivants" définissant le premier manifeste), on ne peut qu'être frappé par le refus répété d'attribuer à l'art dramatique toute possibilité de discours idéologique, alors même qu'il est sans cesse envisagé comme moyen de propagande d'"action". Cette contradiction apparente disparaît si l'on tient compte des nombreuses allusions à la valeur "exaltante" d'un spectacle semi-improvisé et vitalissimo, aux actions aussi "maladroites, réglées ou prévisibles" que la vie elle-même, mais capable "de s'enthousiasmer devant des reflets rouges et verts sur les fauteuils" jusqu'au "dynamisme absolu".

C'est le théâtre dans sa forme même, comme entrainement à l'héroïsme et exercice éthique en soi, qui s'impose finalement dans ces manifestes. Le modelage des âmes juvéniles trouve ici son accomplissement parfait dans la cascade imitative du mouvement frénétique et enthousiaste des éléments primordiaux du réel chaotique ${ }^{15}$ transmis aux Futuristes et à leur public. 

l'éditeur Sansot ${ }^{17}$. Dans cette version italienne de l'ouvrage publiée en 1915 à Milan, aux éditions futuriste de Poesia dirigées par Marinetti en personne, celui-ci reprend les anciens articles, discours et manifestes français publiés en 1910 et 1911, en ajoutant toutefois une série de textes qui assurent des fonctions introductives ou transitionnelles pour ce qui constituera in fine à la fois une histoire de son mouvement et un brulôt interventionniste, en l'inaugurant par l'évocation de la revue littéraire Poesia et en le concluant par les trois manifestes politiques diffusés par son mouvement en 1909, 1911 et 1913.

Très rapidement, les principales modifications apportées par Marinetti pour ce premier essais italien de propagande futuriste consistent en une série de retraits (Ce déplorable Ruskin, Le mépris de la femme, le manifeste de fondation et tous les manifestes présents dans Le futurisme que Marinetti n'avait pas signés), et d'ajouts divers (Lettera futurista ai cittadini di Parma, Battaglia di Trieste et Discorso ai Triestini, Préface et «discours» de Mafarka il futurista, La Divina Commedia è un verminaio di glossatori, Battaglia di Venezia et Discorso futurista ai Veneziani, Contro Roma passatista, Contro l'amore e il parlamentarismo, Nascita di un'estetica futurista, Sintesi della guerra (table mot-libriste), 1915 In quest'anno futurista et les trois manifestes politiques cités).

L'interprétation globale de ces modifications ne semble pas soulever de problèmes majeurs: il s'agissait pour Marinetti essentiellement de mettre de côté des textes aux thématiques trop culturelles ou trop sectorielles pour mieux étoffer la composante nationaliste de son discours, à usage d'un pays qui allait officiellement entrer en guerre le 24 mai 1915, en rappelant aux lecteurs italiens d'une part l'activisme interventionniste du 
mouvement futuriste sur le territoire national (Parme et Rome, en plus de Milan, Turin, Venise ou Trieste irredenta), mais en insistant également, essentiellement auprès des étudiants, sur le bellicisme exacerbé inhérent à son avant-garde, au point d'identifier ce mouvement avec la guerre elle-même. À cet égard, les derniers textes du recueil sont extrêmement significatifs, en ce qu'ils illustrent la polémologie intégrale, à la fois ontologique, éthique, esthétique et politique, du Futurisme.

Après les étonnants Nascita di un'estetica futurista et La guerra elettrica, exaltés et visionnaires, après une table mot-libriste intitulée Sintesi futurista della guerra sur laquelle nous reviendrons dans un instant, 1915 in quest'anno futurista est lui aussi extrêmement révélateur de ce bellicisme métaphysique ${ }^{18}$, tandis que les trois manifestes politiques sont encadrés de courts paragraphes qui en exaltent la violence "ouvertement guerrière et férocement patriotique", quelle soit irredentiste, impérialiste, anticléricale et antisocialiste, antibourgeoise et antiacadémique - en un mot donc, antipasséiste.

Le tout dernier paragraphe du livre, après le rappel de ces tracts et placards politiques "lancés", "publiés", “diffusés" comme le rappelle la voix narrative marinettienne, qui par ailleurs réactualise ces actions en rediffusant une fois encore ces textes, conclut ainsi cette histoire du Futurisme:

Du 20 août 1915 à aujourd'hui, en attentant la joie de combattre à la frontière orientale, nous avons organisé les deux premières manifestations antineutralistes extrêmement violentes de Milan. Nous avons également dirigé, dans les universités et sur les places de Rome et de Milan, plus de 30 manifestations tout aussi efficaces. Nous avons été plusieurs fois arrêtés, et nous avons été les seuls à endurer 5 journées de prison pour avoir exigé avec violence notre grande guerre hygienique.

Tout le récit historique est donc orienté vers la guerre imminente que s'apprète à livrer un narrateur sans cesse hésitant entre io et noi, entre peuples et poètes, entre Place, Université et Frontière: et cette actualisation du passé est encore accentuée par une narration qui, depuis la toute première page de l'ouvrage et malgré les indications chronologiques répétées, ne cesse de raconter cette guerre actuelle et totale futuriste, depuis ses

Premières batailles futuristes

Le 11 octobre 1908, après avoir travaillé pendant 6 ans à ma revue internationale Poesia afin de libérer de ses fers traditionnels et mercantiles le génie poétique italien menacé de mort, je sentis d'un coup que les articles, les poésies et les polémiques ne suffisaient plus. Il fallait absolument changer de méthode, descendre dans les rues, donner l'assaut aux théâtres, et introduire le coup de poing dans la lutte artistique. [...]

Le 20 février 1909 je publiai dans le Figaro le célèbre Manifeste du Futurisme. Ce fut la mèche allumée de notre grande rébellion contre le culte du passé, la tyrannie des académies et la basse vénalité qui écrasent la littérature contemporaine. [...]

Après la victoire futuriste de Trieste, remportée au Politeama Rossetti, nous comparûmes subitement sur la scène du Théâtre Lyrique de Milan [...]

J'avais avec moi de grands et très jeunes poètes. Ils dénoncèrent avec moi, en vers et en prose, l'état ignominieux dans lequel se trouvait notre élite intellectuelle, l'opportunisme et la médiocrité qui dictaient notre politique étrangère et la nécessité urgente de relever à tout prix notre dignité nationale, sans laquelle il n'y a ni art ni littérature possible.

[...] avec toute la force de mes poumons, je criai alors «Vive la guerre, seule hygiène du monde! À bas l'Autriche». Ce cri, répété avec insistance, déclencha une bataille dans la salle tout entière, qui se divisa immédiatement en deux camps. [...]

À Turin, la troisième Soirée futuriste fut une bataille grandiose. ${ }^{19}$ 

réitérée en d'innombrables occasion dans la diégèse et dans la narration de Guerra sola igiene del mondo, que culmine ce qu'il faut bien appeler une histoire sans passé - histoire sans passé dont Marinetti, les futuristes et les jeunes italiens sont les protagonistes indistincts, réunis souvent en un "nous » (noi) aussi fictif que problématique, qui force une lecture déjà partisane, alors qu'en dernière analyse les antagonistes se réduisent comme toujours à un seul, unique et puissant: le passéisme.

et ses villes constituent d'abord l'espace privilégié du récit, un cadre national encore dramatiquement incomplet converti par contagion à l'ivresse futuriste, tandis que progressivement le propos s'élargira à l'Europe et au monde tout entier. L'unité dramatique est cristallisée par un terme unique et récurrent, battaglia, auquel font échos tous les accents et allusions polémologiques du recueil, innombrables. Il n'y a aucun autre référent historique que la guerre, l'incessante guerre contre les morts finalement assimilée à la guerre contre l'Autriche-Hongrie et ses alliés ${ }^{20}$.

Ces techniques narratives et propagandistes seront systématiquement reprises et peaufinées dans Democrazia futurista (1919), Futurismo e fascismo (1924), et Marinetti e il futurismo (1929) dans lesquels, sur le modèle de Guerra sola igiene del mondo, la narration historique tend à disparaître, tout comme la chronologie, occultées par le collage, en guise de citation directe, de textes recyclés et reportés tels quels, qui concernent les aspects les plus divers de la société.

41 Le passé de la narration est ainsi sans cesse interrompu par l'apostrophe, la répétition nécessaire, l'invective, pour disparaitre enfin et laisser place à un présent littéralement explosif, tout entier actualisé dans une temporalité relevant à la fois d'un « Devenir fou » ${ }^{21}$ et d'un propos urgent d'actualité (la guerre, la révolution futuriste, ou bien, après la prise de pouvoir de Mussolini, la revendication dérisoire d'un lien génétique entre futurisme et fascisme). Le présent de Marinetti n'est engendré par aucun déterminisme héréditaire: il apparaît comme une libre génération spontanée, "détaché de tout lien génétique avec le passé" et "prêt à la construction des puissances futures"22; le refus absolu et radical de tout lien historique est compensé par le parti pris déterminé d'une attention tout entière vouée aux formes de vie sociale modernes et à leurs images mystérieuses.

En guise de conclusion, cette histoire sans passé, mais également sans avenir autre qu'utopique, cette guerre totale sans fin contre le passéisme sous toutes ses formes nous semble être représentée, et faire l'objet d'une narration paradoxale et emblématique, dans la table mot-libriste déjà évoquée Sintesi futurista della guerra, qui assimilera de façon fort frappante le combat du Futurisme contre le passéisme à la guerre mondiale des Alliés contre les Empires centraux. L'opération propagandiste est menée visuellement en exploitant l'angle aigu et pénétrant de la volonté (déjà repris par Luigi Russolo dans son huile sur toile de 1911, La rivolta) pour un manifeste en forme de poésie graphique totalement unique en son genre, où la guerre est figée dans un schéma synoptique, mêlant peuples et poètes, qualités éthiques individuelles et jugements culturels nationaux, insultes et dérision de l'ennemi passéiste (sur d'autres variante, l'Empire Ottoman rejoint l'Allemagne et l'Autriche avec un lapidaire: "Turquie $\}=0$ »). L'interventionnisme futuriste se charge ici d'une idéalité toute post-romantique, en créant un manifeste à la fois extraordinaire d'invention et d'humour, et aux nombreuses variantes d'une guerre redoutable d'implications artistiques, idéologiques et politiques ${ }^{23}$.

Cahiers de Narratologie, 15 | 2008 
Futurisme, avant-garde, idéologie des avant-gardes, Marinetti, manifestes futuristes Italie

L'article examine le refus du genre historique en tant qu'expression artistique viable par le Futurisme italien, en revenant sur les manifestes et les essais qui établissent un rapport entre le projet avant-gardiste des années Dix et Vingt et ce genre littéraire et théâtral très populaire à l'époque. Le rapport à l'histoire et au passé constitue en effet un problème inhérent à la naissance de l'avant-garde futuriste : ainsi, nous examinons ici l'élaboration de la notion de " passéisme ", mais également la métaphore de la guerre aux morts, toutes deux stratégiques dans la constitution idéologique du mouvement. Enfin, nous revenons sur les essais de F.T. Marinetti écrits entre 1915 et 1929 dans lesquels le genre narratif où domine la référence historique est paradoxalement prééminent, et en particulier sur Guerra sola igiene del mondo, publié en 1915.

L'articolo si propone di esaminare il rifiuto del genere storico in quanto forma artistica praticabile da parte del Futurismo, tornando sui manifesti che stabiliscono un rapporto tra il progetto avanguardista degli anni dieci e venti e questo genere letterario e teatrale molto in voga all'epoca. In effetti, il rapporto alla storia e al passato costituisce un problema inerente alla nascita stessa del Futurismo. Si esamineranno dunque l'elaborazione della nozione di "passatismo" e della metafora della guerra contro i morti, ambedue strategiche nella costituzione ideologica del movimento marinettiano. Infine, si tornerà brevemente sui saggi pubblicati da Marinetti tra il 1915 e il 1929 (e in particolare sul volume Guerra sola igiene del mondo, del 1915) nei quali il genere narrativo preeminente è paradossalmente proprio quello storico.

\section{SINTESI FUTURISTA DELLA GUERRA}

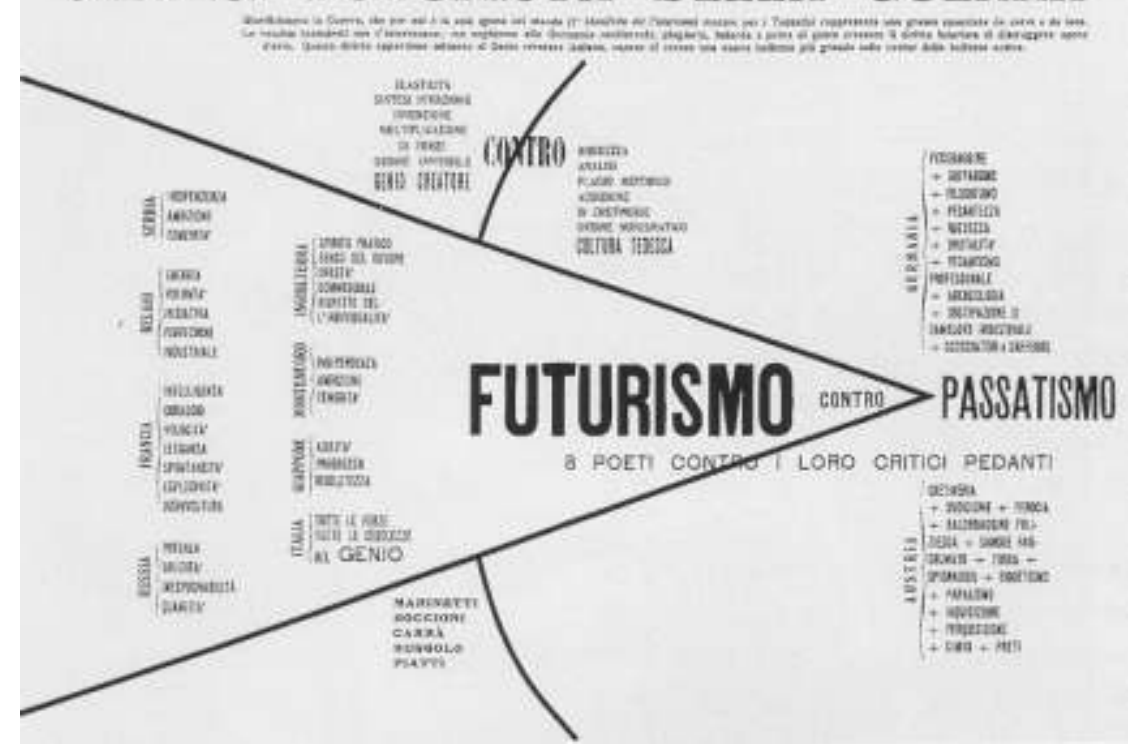

F.T. Marinetti, U. Boccioni, C. Carrà, L. Russolo, U. Piatti, Sintesi futurista della guerra, 1914. Droits réservés. 


\section{NOTES}

1. U. BOCcIONI, « Il cerchio non si chiude », in Lacerba, a. II, $n^{\circ} 5$, Florence, $1^{\mathrm{er}}$ mars 1914.

2. G. PAPINI, « Il passato non esiste », in Lacerba, a. II, n² 2, Florence, 15 janvier 1914.

3. F.T. MARINETTI, Fondazione e manifesto del futurismo, 1909. Cette version du texte inclut des passages absents de la première version parue dans le Figaro (pour une comparaison des versions successives du manifeste de fondation, on consultera Le premier manifeste $\mathrm{du}$ futurisme, édition critique avec en fac-similé le manuscrit original de F. T. Marinetti, édité par JP. A. de Villers, Ottawa, Editions de l'Université d'Ottawa, 1986, ainsi que l'anthologie éditée par E. Caruso, Manifesti, proclami, interventi e documenti teorici del futurismo, Florence, Spes-Salimbeni, 1980, pour tous les autres manifestes cités).

4. Lettre circulaire aux journaux, rédigée en français non datée et non signée, 1910, in F.T. MARINETTI, Le futurisme, Paris, Sansot, 1911 (réédité par G. Lista, Lausanne, L'Age d'Homme, 1980, p. 89). C'est dans le Dernier chant du pèlerinage d'Harold, publié en 1825 et consacré à Byron, que Lamartine écrit que l'Italie est « la terre du passé [...] un monument écroulé », habité par « la poussière humaine [...] l'ombre d'un peuple » : il suivait alors notamment Mme de Staël, qui avait écrit dès 1807 qu'à l'Italie, « patrie des tombeaux », ne restait plus que « la gloire de ses morts » (in Corinne ou l'Italie). En fait, l'expression « terre des morts » apparaît telle quelle chez Lamenais, Anatole France, Gaston de Pawlowski, Paul Bourget ou Maurice Barrès, mais pas toujours avec le même mépris que chez Lamartine, qui fut défié en duel (et blessé au bras, puis noblement soigné) par Gabriele Pepe, en exil, pour avoir offensé l'Italie. Giuseppe Mazzini écrivait pour sa part, en réponse à ce topos littéraire très français, mais pas seulement : « C'est si beau une nation morte : moins inquiétant, de toute façon, qu'une nation qui souffre ", in Scritti letterari di un Italiano vivente, 1847. Enfin, Marinetti et Boccioni reprennent encore polémiquement cette expression (cf. Manifeste de Fondation et Pittura e scultura futurista), et La Terra dei vivi sera enfin le titre d'une revue futuriste de La Spezia, dirigée par Fillìa de juin à octobre 1933.

5. U. BOCCIONI, C. CARRÀ, L. RUSSOLO, G. BALLA, G. SEVERINI, Manifesto dei pittori futuristi, 1910.

6. Ibid.

7. F.T. MARINETTI, Contro Venezia passatista, 1910; ce texte inaugurait également des techniques propagandistes qualifiées à l'époque d' "américaines», et rejetées par le monde littéraire et artistique, avec distributions de placards en nombre et actions spectaculaires devant les foules urbaines médusées. Cf. la préface de Palazzeschi à Teoria e invenzione futurista, , édité par L. De Maria, Milan, Arnoldo Mondadori, « I Meridiani », 1968; cf. également le commentaire édifiant qu'en fera Marinetti lui-même in Guerra sola igiene del mondo («La battaglia di Venezia»), repris également in Teoria e invenzione futurista, cit., p.

268.

8. On pourra consulter à cet égard l'anthologie de textes des voyageurs français aux XVIII e et XIX ${ }^{\mathrm{e}}$ siècles éditée par Y. Hersant, Italies, Paris, Robert Laffont, 1988, souvent réédité.

9. C'est Nietzsche précisément, avec la Considération Inactuelle intitulée De l'utilité et de l'inconvénient des études historiques pour la vie, qui est à la source de l'anti-passéisme marinettien naissant. Le texte, écrit en 1874, est traduit en français en 1907.

10. F.T. MARINETTI, La voluttà di essere fischiati, in Guerra sola igiene del mondo, 1915. 
11. natura est à entendre au sens de Boccioni, tel qu'il est explicité dans la citation cidessus : un phonographe (pour reprendre un autre exemple boccionien) au même titre que les arbres, les fleurs et les machines: tout cela est natura, et relève d'un devoir de (re)création de milieu favorable à la nouvelle humanité.

12. F.T. MARINETTI, E. SETTIMELLI, B. CORRA, Il teatro futurista sintetico, 1915. La première partie de cet extrait n'avait pas été publiée en français, alors que la seconde partie du texte avait été traduite et diffusée par Marinetti lui-même, très probablement. Celui-ci avait, comme Severini, puis Soffici, Papini ou Prampolini, une politique propagandiste très attentive à la vie culturelle parisienne, mais comme on pourra le vérifier encore ci-dessous, les textes ou paragraphes interventionnistes étaient le plus souvent réservés à une diffusion strictement italienne.

13. Cf. F.T. MARINETTI, F. CANGIULLO, Il teatro della sorpresa, 1921; F.T. MARINETTI, Dopo il teatro sintetico e il teatro a sorpresa, noi inventiamo il teatro antipsicologico astratto di puri elementi e il teatro tattile, 1924; F.T. MARINETTI, Il Teatro Futurista Aereoradiotelevisivo, 1931; F.T. MARINETTI, Il teatro totale, 1933.

14. E. PRAMPOLINI, L'atmosfera scenica futurista - Manifesto tecnico, 1932; cf. également Scenosintesi plastica dinamica, 1927; L'atmosfera scenica futurista, 1924. Pour ce qui est du théâtre futuriste, on consultera également les anthologies Théâtre futuriste italien: anthologie critique, édité par G. Lista, Lausanne, l'Age d'homme, 1976, et Teatro del tempo futurista, édité par M. Verdone, Roma, Bulzoni, 1988.

15. Rappelons que Il teatro futurista sintetico propose un paragraphe cosmologique fondamental.

16. Marinetti considérait son mouvement comme d'extrême gauche, ainsi qu'il l'écrivit à plusieurs reprises aussi bien dans les années Dix que dans les années Trente.

17. MARINETTI F.T., Le futurisme, Paris, Sansot, 1911. Premier essai futuriste, ce volume était destiné « Aux étudiants de Paris, pour qu'ils aiment la grande Italie futuriste que nous préparons ". Il contient donc les textes qui seront traduits et parfois légèrement modifiés en 1915 dans Guerra sola igiene del mondo: Les premières batailles; Discours futuriste aux Anglais; Ce déplorable Ruskin; Trieste, notre belle poudrière; La guerre, seule hygiène du monde; Le mépris de la femme; L'homme multiplié et le règne de la Machine; Nous renions nos maîtres symbolistes, derniers amants de la lune; Ce qui nous sépare de Nietzsche; La volupté d'être sifflé; $L a$ Guerre électrique. Le volume contient également les premiers manifestes et proclamations futuristes: Premier manifeste du Futurisme, Tuons le Clair de Lune!, Manifeste des Peintres futuristes, Manifeste des Musiciens futuristes, Premier Manifeste futuriste aux Vénitiens et Proclamation futuriste aux Espagnols, enfin une conférence de Marinetti à la Maison des étudiants (9 mars 1911) et une interview à J. Bois, de Le Temps (14 mars 1911).

18. À propos de ce manifeste, on pourra consulter notre article « Mythe et violence : Georges Sorel dans les manifestes du Futurisme italien ", in Cahiers de Narratologie n 14, CIRCPLES - REVEL, Nice, février 2008. ( http://revel.unice.fr/cnarra/document.html?id=634 ) .

19. F.T. MARINETTI, «Prime battaglie futuriste », in Guerra sola igiene del mondo, Milan, Edizioni futuriste di Poesia, 1915.

20. Cf. Serge Tchakhotine, Le viol des foules par la propagande politique, 1939 (réédition Paris, Gallimard « Tel », 1992), où la propagande est définie comme l'association d'un thème idéologique à une pulsion instinctive, avec des caractéristique ici déjà présentes: simplification et ennemi unique, grossissement et défiguration, orchestration, répétition, transfusion de mythes préexistants, recherche d'unanimité et de contagion. 
21. Cf. F.T. MARINETTI, Lettre ouverte au futuriste Mac Delmarle, 1913, et Lo splendore geometrico e meccanico e la sensibilità numerica, 1914.

22. F.T. MARINETTI, Prefazione futurista a Revolverate di Gian Pietro Lucini, 1909.

23. F.T. MARINETTI, U. BOCCIONI, C. CARRÀ, L. RUSSOLO, U. PIATTI, Sintesi futurista della guerra, 1914. Droits réservés (la version présentée diffère très légèrement de celle du 20 septembre 1914 reproduite dans Guerra sola igiene del mondo). Pour une analyse des caractéristiques des tables mots-libristes futuristes, cf. Tavole parolibere futuriste. Antologia 1912 - 1944, édité par L. Caruso et S. M. Martini, Naples, Liguori, 1977.

\section{RÉSUMÉS}

L'article examine le refus du genre historique en tant qu'expression artistique viable par le Futurisme italien, en revenant sur les manifestes et les essais qui établissent un rapport entre le projet avant-gardiste des années Dix et Vingt et ce genre littéraire et théâtral très populaire à l'époque. Le rapport à l'histoire et au passé constitue en effet un problème inhérent à la naissance de l'avant-garde futuriste: ainsi, nous examinons ici l'élaboration de la notion de "passéisme", mais également la métaphore de la guerre aux morts, toutes deux stratégiques dans la constitution idéologique du mouvement. Enfin, nous revenons sur les essais de F.T. Marinetti écrits entre 1915 et 1929 dans lesquels le genre narratif où domine la référence historique est paradoxalement prééminent, et en particulier sur Guerra sola igiene del mondo, publié en 1915.

L'articolo si propone di esaminare il rifiuto del genere storico in quanto forma artistica praticabile da parte del Futurismo, tornando sui manifesti che stabiliscono un rapporto tra il progetto avanguardista degli anni dieci e venti e questo genere letterario e teatrale molto in voga all'epoca. In effetti, il rapporto alla storia e al passato costituisce un problema inerente alla nascita stessa del Futurismo. Si esamineranno dunque l'elaborazione della nozione di "passatismo" e della metafora della guerra contro i morti, ambedue strategiche nella costituzione ideologica del movimento marinettiano. Infine, si tornerà brevemente sui saggi pubblicati da Marinetti tra il 1915 e il 1929 (e in particolare sul volume Guerra sola igiene del mondo, del 1915) nei quali il genere narrativo preeminente è paradossalmente proprio quello storico.

\section{INDEX}

Mots-clés : avant-garde, Futurisme, idéologie des avant-gardes, Marinetti, manifestes futuristes

\section{AUTEUR}

\section{SERGE MILAN}

Université de Nice-Sophia Antipolis, CIRCPLES, EA 3159 